\title{
Author Index and Subject Index
}

(c) The Korean Institute of Metals and Materials 2021

The online version of this article contains the Author index and Subject index as supplementary information

Supplementary Information The online version contains supplementary material available at https://doi.org/10.1007/s12540-021-01134-z.
Publisher's Note Springer Nature remains neutral with regard to jurisdictional claims in published maps and institutional affiliations. 